\title{
Seamless Prevention of Adverse Events from Tattooing: Integrated Strategy Emphasising the Customer-Tattooist Interaction
}

\author{
Jørgen Serup \\ Bispebjerg University Hospital, Department of Dermatology, the 'Tattoo Clinic', Copenhagen, Denmark
}

\begin{abstract}
The boom in tattooing has been paralleled by more frequent adverse events, which may be localised in the skin or systemic and manifested clinically or latent. Infections, allergic reactions from red-coloured tattoos and papulonodular reactions from black tattoos dominate. Mild complaints are very common, with $1 / 5$ of all tattooed individuals having acquired sensitivity to sunlight in the tattooed skin. The potential risk of cancer due to potential carcinogens in some tattoo inks has hitherto not manifested in clinical reports, despite the millions of people who have been tattooed over many decades. A risk of death from tattooing remains associated with severe infection, i.e. sepsis. Preventive strategies may rely on focused preventions, and sterility and preservation of ink is essential, rational and knowledge-based. The chemical and particle contents of ink nanoparticles cannot be unrestricted; however, focused control of ink is facing many uncertainties, including analytical problems, lack of identification of allergens in ink and discrepancies between the content of potential carcinogens and manifestation of cancer in the clinic. The concept of seamless preven-
\end{abstract}

tion is introduced as a pragmatic strategy that emphasises the customer-tattooist interaction, which is the 'engine' of tattoo safety. This strategy amalgamates the range of narrow-scope preventive instruments and shall ensure that any relevant instrument is used actively and without deficiency or drop out, thus resulting in a complete orchestration of a multi-targeted strategy. High-priority elements of this strategy shall facilitate a qualified 'go' or 'no go' decision by the customer before the tattoo is made and should involve informed consent, qualification of the tattooist and the parlour, including supplies of inks etc., and attention to hygienic security. Records and documentation of tattoo cases with complications and the culprit inks as well as the establishment of national or European-based surveillance systems that are properly equipped to deliver efficient clarification and handling of adverse events and hazards of tattooing and inks, which needs attention and timely action to prevent additional cases and epidemic outbreaks, are part of this seamless strategy, along with optimised medical therapy and research.

(c) 2015 S. Karger AG, Basel 


\section{Introduction}

Tattooing is, with some 100 million Europeans being tattooed, the largest on-going experiment involving the injection of chemicals and microparticles into the skin and body of man. Tattooing is an old art and is felt to belong to the roots of the people; thus, it is culturally difficult to approach this issue with authoritative restrictions. It is already solidly established in societies and has an established culture of its own. People may feel that they are within their democratic and human rights to exert ownership of their own skin and decide to run the risk that comes with the satisfaction of putting their personal signature on their skin.

However, from a toxicological and medical point of view, the practice of tattooing is an obvious risk to health. The scattered regulation of tattooing and inks in countries is out of proportion with the world-wide heavy regulation of pharmaceuticals, in which the new registration and international launch of just one new drug with just one pure chemical requires an investment on the order of a billion dollars.

Nevertheless, it shall be recognised that tattooing, despite its widespread use all over the world and the number and occurrences of tattoos, has caused remarkably few identified medical complications and alerts. However, the development of the global tattoo business is dynamic and involves the constant introduction of new practices, tattoo ink ingredients, contaminants and products, as well as the risk of adverse events and hazards that present an ever-lasting threat to health.

This chapter launches the concept of the 'seamless prevention' of tattoo adverse events, as worked out in more detail in a recent report of the Council on Health and Disease Prevention, which is incorporated with the Danish Medical Association [1]. This concept is pragmatic and attempts to face facts and reality for the fulfilment of its aims, while understanding the necessity of the ac- ceptance and partnership with stakeholders in order to become implemented and truly improve the situation.

\section{Tattoo Risks, Aims and Targets of Prevention}

Tattoo complaints and complications and their diagnosis and spectrum are described in a separate chapter of this book and in a number of reports and reviews in the medical literature [27].

In brief, the observed adverse events, which are candidate objects of prevention, are described below.

The Known Risks of Tattooing

Medical complications and interactions

- Bacterial infections manifested as acute infections after tattooing and during the course of healing of the tattoo. These may be local or systemic and ultimately progress to sepsis.

- Traumatic and other local skin events due to improper tattoo technique and amateurism, with acute, subchronic or chronic consequences.

- Chronic infections introduced with the tattoo, especially viral hepatitis B and C and HIV infections.

- Chronic allergies originated from the ink, especially those occurring in tattoos with red or shades of red, which are supposed to be somehow associated with azo-pigments but are also occasionally seen in green and blue tattoos.

- Chronic papulo-nodular reactions in black tattoos that are supposed to be associated with pigment overload and agglomeration inside the skin that happens spontaneously over time.

- Special chronic reaction patterns that are, to examplify, associated with the lymphatic system and pain syndromes that are associated with the sensory nervous system. 
- Interactions with skin diseases and skin conditions, generally and specifically, and interactions with general diseases and weaknesses of the body.

Mild complaints

- Intermittent or constant itch and swelling, with discomfort and photosensitivity; the latter is observed in 1/5 of tattooed individuals $[8,9]$.

Regret of tattoos, psycho-social consequences and tattoo removal by lasers

- Regret of a tattoo is frequent.

- Regret of a tattoo and psychic and social complications, which may be severe, limit life and block opportunities.

- Removal of a tattoo using laser or other methods resulting in scar formation, abnormal melanin pigmentation, allergic reaction or other complications. Many tattoos cannot be removed efficiently with an aesthetically acceptable result and without scarring using lasers.

Life-threatening and exceptional hazards

- Bacterial infection with sepsis, multiple organ failure and death, by incident.

- Anaphylactic allergic reactions, with the allergen released during laser treatment, or latex protein allergy induced by the tattooist's gloves, which is exceptional.

- Syncopes during tattooing due to a new or pre-existing medical disease or condition, i.e. cardiac problems and arrest.

\section{The Potential Risks of Tattooing}

- Manifestations of carcinogenic, mutagenic and reproduction-toxic (CMR) ingredients of inks and manifestations of the toxic or allergic effects of metals. Risks remain potential since clinical reports and epidemiological data have not confirmed such risks to be clinically significant with the use of present tattoo inks.

- Manifestations related to pigments which, as nanoparticles and microparticles, might exert hitherto unknown effects in the body;

however, these are presently not a subject of specific study or reporting.

The list of isolated risks and scenarios of risk related to tattooing uncovers a broad range of mechanisms, unwanted effects and safety end points, which prevention shall ideally address. The list is exhaustive, and prevention as a whole goes far beyond the narrow regulation of tattoo inks and hygiene. The more common adverse events shall be the primary aims of prevention. Sepsis is uncommon but remains the primary reason for death, unless treatment is efficient. Severe infection has for more than a century been the main cause of severe disability, amputations and deaths from tattooing [10].

\section{State of the Knowledge and Need for Research as a Back-Up for Preventive Measures}

The incidence and prevalence of tattoo complications have not been studied systematically, and prevention has to rely on clinical and epidemiological evidence that has accumulated over the years and is reported in the medical literature by professionals of different backgrounds who may be confronted with such events. A search of the National Library database for medical reports (as of 15 November 2014, PubMed) resulted in the hits that are shown in table 1.

The literature includes many individual case reports and may provide limited evidence; however, the figures support the impression that allergic reactions and infections are prominent and that red and black tattoos, which are the more popular colours, generate more frequent reports. Tattoo problems are clearly underreported [11]. Since its start in 2008, the 'Tattoo Clinic' of Bispebjerg University Hospital has collected some 400 cases of tattoo complications from the Copenhagen major region. The EU Rapid Alert System for non-food (RAPEX) products has very few reports 
Table 1. Incidence and prevalence of tattoo complications illustrated from the National Library database, index words and hits

\begin{tabular}{lr}
\hline Tattoo & 1,533 \\
Tattoo reactions & 158 \\
Tattoo complication & 48 \\
Tattoo allergy & 133 \\
Tattoo red & 134 \\
Tattoo blue & 67 \\
Tattoo green & 46 \\
Tattoo yellow & 22 \\
Tattoo black & 166 \\
Tattoo infection & 186 \\
Tattoo sepsis & 5 \\
Tattoo laser & 289 \\
Tattoo laser removal & 188 \\
Tattoo cancer* & 244 \\
Tattoo lymph node & 51 \\
Skin cancer & 146,473 \\
\hline
\end{tabular}

* Includes black tattoos used for medical purposes, i.e. marking of the radiation field in breast cancer.

of problematic tattoo inks and cannot contribute significant information on the clinical adverse reactions associated with tattooing because it is not a clinical database.

The reports of cancer from tattoos are consistent with the recent review of the world medical literature as searched by Kluger and Koljonen [12]. These authors checked the reports and traced only 50 valid cases of cancers (including keratoacanthoma, a dubious 'cancer' that is benign in clinical development but shares features with squamous cell carcinoma by histology) arising in tattoos. They concluded that these cancers likely originated by coincidence, since cancers may easily arise in the epidermis, independent of tattoo pigment. This conclusion is in agreement with two studies of 25-year follow ups of patients with psoriasis and atopic dermatitis who were repeatedly treated with tar on large areas of their skin over several weeks, sometimes combined with ultraviolet-B light (i.e. the Goeckerman regimen) $[13,14]$. Follow up showed no increase in skin cancer. The skin organ, in contrast to airway epithelium and lung tissue, appears to be remarkably resistant to the development of cancer from tar, which is rich in polycyclic aromatic hydrocarbons (PAHs). This contrasts data from the chemical screening of tattoo inks, which found PAHs and primary aromatic amines (PAAs) in the inks, but apparently these do not cause clinical cancer in tattooed skin [15]. Today, tar treatment is accepted and used as an efficient treatment of psoriasis and eczema in dermatology, used as 2nd- or 3rd-line treatment because of its unpleasant smell and staining.

The preventative measures for skin cancer due to tattooing and ink ingredients, which are based on register data on potential carcinogenicity, is not applicable in this situation since such cancers in the tattooed skin, regional lymph nodes and other organs apparently do not occur under reallife conditions. What is not present, of course, cannot be reduced through prevention.

It shall, nevertheless, be noted that the adverse effects of CMR substances in tattoo inks that manifest in any organ can not be academically excluded, particularly reproduction-toxic effects, when a single dose of a mutagen at a critical stage of cell division might potentially cause harm.

It shall also be noted that tattoo inks cannot acceptably have unlimited amounts of CMR substances; therefore, there is an urgent need for some regulatory limitation, if not based on conclusive research then, as a minimum, shaped as a 'soft' regulation.

With respect to allergic reactions to tattoos, especially to tattoos using red and shades of red, it was demonstrated in a large study involving allergy-patch testing of ninety patients that patients who had allergic reactions did not react to a chemical or allergen from the specific culprit tattoo ink stock bottle and thus had not reacted to the free PAAs in the products [16]. Allergic tattoo reactions typically occur after weeks, months or years and are thought to occur due to a hapten that has formed in the tattooed skin by local metabolism. 

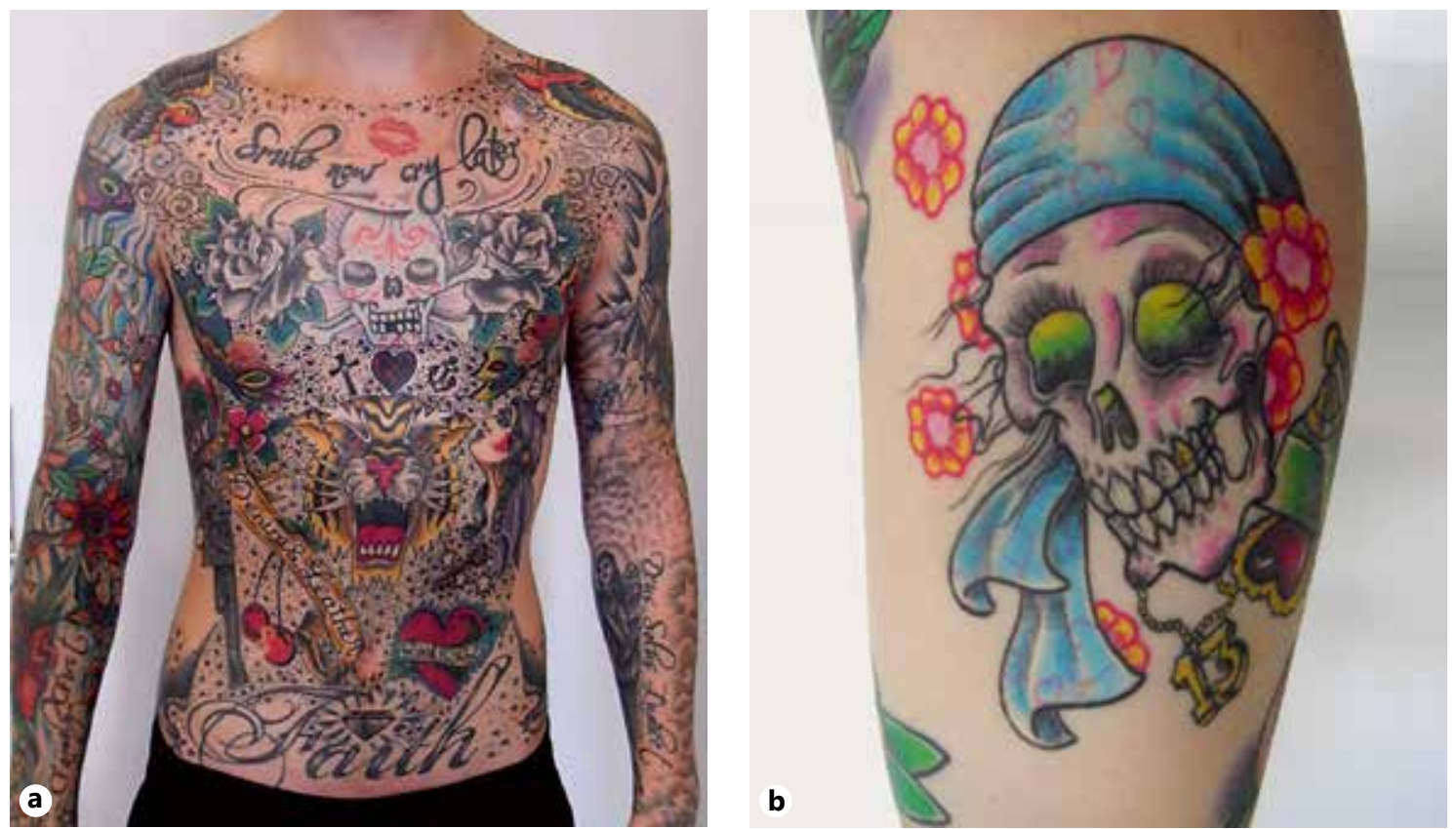

Fig. 1. a Extensively tattooed young man. The tattoo shown in the photo covers $27 \%$ of the person's skin, i.e. about $0.5 \mathrm{~m}^{2}$ or $5,000 \mathrm{~cm}^{2}$, according to 'the rule of nine'. However, the extensive skin fields inside the frame of the tattoo are not completely tattooed, and about 10 different colours were used at varying intensity to create shades. The dose of each colour per $\mathrm{cm}^{2}$ is much lower than the outline of the tattoo and is very difficult to estimate unless sophisticated computer technology is used to assess the area. The toxicological risk is assumed to be colour-specific. Thus, the simple measurement of the size of a tattoo is imprecise when measuring the area-wise exposure to tattoo ink of a defined colour. $\mathbf{b}$ The same problem illustrated for a tattoo on the shoulder of a young woman.

The hapten, epitope and substrate raw material in tattoo ink are presently unidentified and thus cannot be analysed, delineated or eliminated from inks. The substrate of allergic reaction to azochemical is not simply a PAA or paraphenylene diamine, as shown in studies on allergic reactions to textile dyes.

Prevention based on the elimination of dangerous ingredients of tattoo inks suffers from a lack of fundamental knowledge, especially on dosage and biokinetics. The local dose of ink in the skin and the absorption, distribution, metabolism and excretion of soluble and insoluble agents, i.e. ingredients and pigment particles, is not understood (fig. 1a, b). Soluble and insoluble ingredients that may slowly release chemicals over years are likely to have very different kinetic profiles and thus very different exposure scenarios. This field is massively under-researched. The translation of register data on PAHs and PAAs and cancer risk in humans is not correlated. Cancer from tattoos appears to not be a problem. The sensitisation potential of isolated PAAs in inks, as mentioned, has not been confirmed, although red ink containing azo chemicals appears to be associated with clinical tattoo allergy. However, the raw material ingredient in ink and the haptens that stimulate allergic reactions are unknown.

The limited knowledge of the aetiology, mechanism and manifestations of tattoo complications calls for pragmatic strategies for the realistic prevention of tattoo complications. 
In contrast to the risks of chemical ingredients and pigment particles, the potential risks of infectious complications are well understood with respect to clinical manifestations, the spectrum of causative microorganisms and the routes of infection. These ingredients can be modified as part of a preventative effort. This rationale is behind infection control well established and universally accepted $[17,18]$. Some part of the microbial risk is attributed to contaminated tattoo ink products, which may include about $10 \%$ of new inks [19, 20]. This threat to tattooed individuals obviously needs to be addressed through development of hygiene policies and guidance for the tattoo parlours.

In conclusion, an overwhelming problem for preventive strategies is that the translation of the potential risk of the chemical and particle contents of tattoo inks, based on register data, into clinical risk to humans is presently poorly understood, premature, limited in predictive value and backed up by little research and validation. This lack of knowledge even includes essential knowledge, such as the nomination of valid standard methods for the chemical analyses of risk elements, which is a key prerequisite for the establishment of meaningful threshold levels of chemical risk elements that are allowed or forbidden in commercial tattoo inks.

\section{Society, Culture and Tattooing in the Perspective of Prevention}

Tattooing is in a boom that has been promoted by media and celebrities. During the last decades, tattooing has propagated from the lower social classes and has become diffusely absorbed by all social segments. Tattooing has become gender neutral and widespread, and populations other than youngsters and gangsters are now tattooed [21]. With tattoo prevalence in the range of $10-20 \%$ of adults and about $30 \%$ of youngsters, prevention is facing a difficult task and a physical barrier due to the size and power of the tattooed population. This development has been paralleled with the opening of a huge number of professional tattoo parlours and even more amateur tattooists, who uncritically buy cheap machines and inks (fig. 2). Furthermore, in modern society, communication using images, which is a modern language that is competing with traditional textual communication, has become mainstream, resulting in massive cultural pressure on individuals in the direction of being tattooed and having personal images and signatures on the skin. On the other hand, the efficient modern society has posed massive pressure and conformity standards on citizens' private lives, limiting their individualism and freedom. Preventive measures limiting the risk of tattooing shall operate in this environment of contrast and incongruence and is bound to have a weak position from the start simply due to the magnitude and popularity of the tattoo business. Any preventive measure must have rationale and concordance with the situation in society to become efficient. Unpopular, authoritative regulation may require extensive control and policing functions to come into operation, which risks the vanishing of the regulations and their ending as a failure, making the construction of such regulations a 'suicide mission'. Tattooing has maintained a rebellious spirit and, even today, is non-obedient in nature and is a signal of setting orders and tight standards aside and saying 'I am my own'.

\section{The Customer-Tattooist Interaction}

The customer-tattooist interaction and mutual confidence described in other chapters of this book appears powerful and important in a preventive perspective. The customer starts with curiosity about tattooing and, under the influence of the surrounding society and his personal environment, friends and close relatives, he or she may decide to contact a tattooist. The tattooist may modify or propose the desired tattoo and in- 
Fig. 2. Low-cost beginner tattooist's kit that is popular among amateurs, supplied with a few disposable needles. The cost of the kit is about EUR 75. The inks named 'Tattoo' are, according to the label, produced in Taiwan, but there is no information on the ingredients or identity of the manufacturer; the origin and legal responsibility cannot be traced.

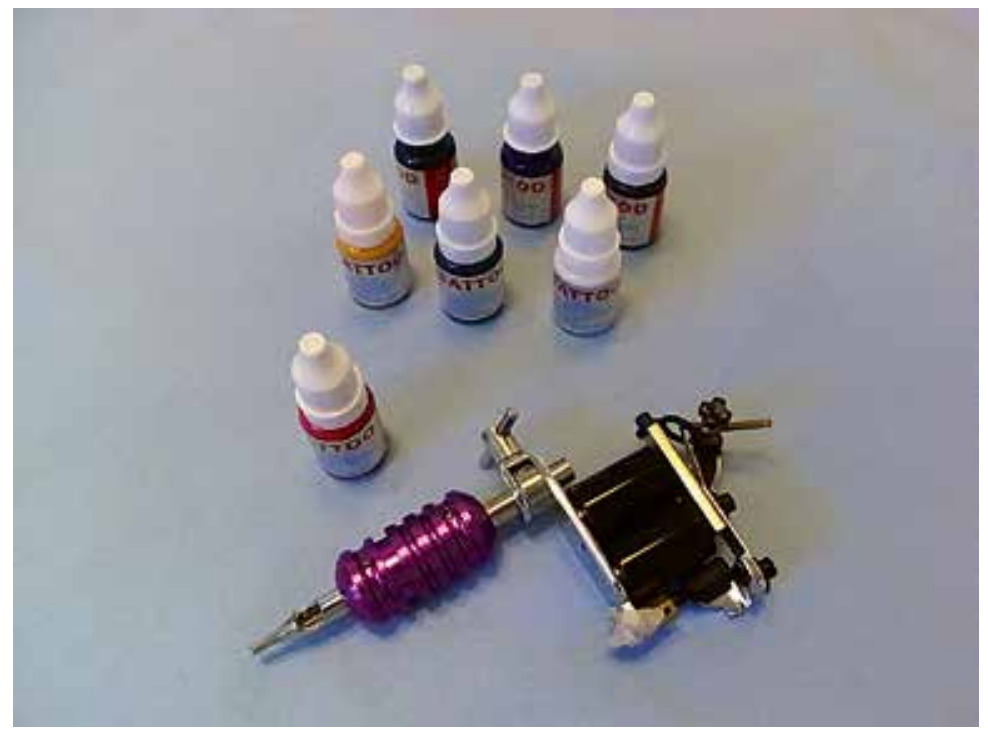

struct the customer, or not, on the risks of tattooing. This is mostly well-accepted, since the customer wishes that his private and permanent signature on his own skin be unique and a mark for life. In that situation, the customer may need to explain the details of his life to the tattooist. This interaction resembles patient-doctor or clientlawyer interactions, and this is a situation when the tattooist may influence a customer's decision on the type of tattoo, its size, location on the body, motive, choice of colour, etc. in a direction that poses more or less risk to the customer, ideally in a spirit of ethical tattooing and high professional standards. The customer-tattooist interaction is the engine of physical tattooing, customer satisfaction and the associated health risk. The tattooist can, as many other professionals, be requested to perform his role up to the requirements given by the society and guidelines from associations of professional tattooists that define professional standards. The job of the tattooist involves the practical execution of the decision to get a tattoo, and for that part, the physical requisites, such as a clean parlour, hygienic instruments and the utilities that should be used. The tattooist should also be educated and experienced. Of course, the ink should be of high quality so that its quality can be ensured and it poses minimum risk; any risk from the ink should be predicted and guaranteed by the manufacturer and distributor. The customer-tattooist interaction also includes advice on aftercare and support in case healing does not occur smoothly. This interaction is of major importance in a preventive perspective because it is individual and because the very act of being tattooed at any time enters a zone of physical risk.

\section{The Supply Industry}

The supply industry comprises manufacturers of tattoo machines, tattoo needles, tattoo ink stock products and other supplies. The supply industry sells their products internationally via local or regional suppliers and via the internet. Any layperson can buy the products. The market is competitive, and the prices of the machines, needles and ink are generally low. American as well as European industries appear to be the leading suppliers of machines and inks. In Europe, tattoo 
inks are only produced in some countries, especially the UK, Germany and Italy, and the majority of European countries have no national production. Inexpensive machines and inks from Asia are important players in the market that help to keep prices low. The market has no obvious room for an increase in the price of supplies. The market is thus price sensitive and appears to have little room for higher price justified by claims of improved safety.

\section{Focused Preventative Strategies}

A focused strategy of prevention targets a selected issue or a sequence of events of major importance in a risk panorama and may act as a key that can lock a door to a disease outcome (by negative listing) or open a door to a safe outcome (by positive listing). Regulation based on specific requirements regarding the chemical composition of tattoo inks, as specified by $\operatorname{ResAp}(2008) 1$ of the Council of Europe, represents a focused strategy that operates by negative listing [22]. To address the real problem, i.e. clinical complications arising from tattooing, the strategy must be essentially rational and thus knowledge based, deemed suited to help the real problem, and be possible to implement, not only sporadically but also coherently in a larger territory, since the tattoo business is international. A critical point is its acceptance by stakeholders and the market. From the start, focused regulation of the negative listing type is positioned weakly when held against the size and nature of tattooing and the tattoo business. If not acceptable, the regulation runs major risk of being neglected and ultimately creating an even more chaotic situation in the market. ResAP(2008)1, originally introduced as a resolution in 2003, regulates the amounts of PAHs, PAAs, metals and microbes in inks, with sterility of inks being a fundamental requirement. The resolution did not manage to become generally accepted in Europe. In countries where the resolution was implemented, follow up showed that inks with PAHs, PAAs and metals had become less frequently found on the shelves of tattoo parlours upon monitored visits and examinations. In addition, the percentage of inks that were contaminated with bacteria decreased. However, per definition unsafe inks were far from eliminated, and there is no indication or evidence that the registered minor change of ink status was accompanied by any reduction in complications from tattooing. No European country (except, very recently, France) has installed a surveillance system to monitor clinical adverse events from tattooing.

ResAP(2008) 1 operates only by the negative listing of chemicals and thus faces firm obstacles to being accepted and implemented. Therefore, stakeholders will need advice on alternative routes. A positive listing of acceptable ingredients in tattoo inks is an obvious and critical need if focused regulation of chemical composition of inks shall ever be successful.

As mentioned above, the rationale behind the focused regulation of microbial contamination of tattoo inks is clear, and since tattoo infections are relatively common and can potentially cause death, this is both a logical and obvious need that can be argued, insisted on and finally accepted. The regulation of microbial contamination of inks may benefit from being separated from the regulation of the chemical content of inks, and from focusing specifically on the sterility and preservation of products. The elimination of microbes in inks and the improvement of hygiene in parlours are synergistic and independent of chemical content.

\section{Integrated/Seamless Prevention Strategy in Tattooing}

In the seamless prevention strategy, a holistic view is taken that recognises the actions of the tattoo customer over time and attempts to influ- 
PUBLIC INSTITUTIONS AND PUBLIC SERVICES

$\begin{array}{llll}\begin{array}{l}\text { Registration of tattooists, parlours } \\ \text { and the legal responsibility }\end{array} & \begin{array}{l}\text { Inspection, approval and } \\ \text { control of parlours }\end{array} & \begin{array}{l}\text { Education of tattooists } \\ \text { and certification }\end{array} & \begin{array}{l}\text { Tattoo regret and } \\ \text { laser removal }\end{array} \\ \begin{array}{l}\text { Treatment of complications by } \\ \text { the public health sector }\end{array} & \begin{array}{l}\text { Disease statistics/surveillance } \\ \text { and general prevention }\end{array} & \begin{array}{l}\text { Tracking individual } \\ \text { events re. causality }\end{array} & \begin{array}{l}\text { Actions to prevent } \\ \text { new hazards }\end{array}\end{array}$

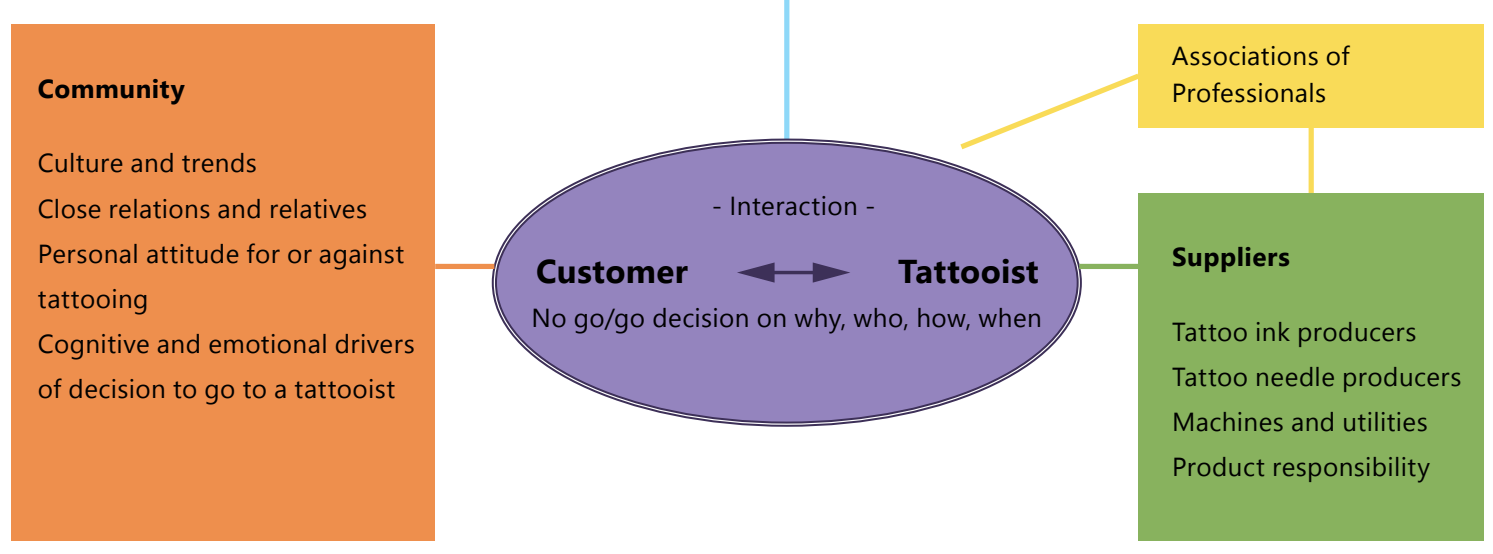

Fig. 3. The seamless prevention model shown as a schematic drawing. The customer-tattooist interaction is essential and the 'engine'. The customer is influenced by society and personal relationships, and the tattooist is influenced by suppliers and professional associations. The suppliers include manufacturers of needles, machines, inks and other utensils. The activity is performed within a legal framework with regulations, guidance, detailed control and surveillance. The responsible society provides medical service and health statistics, and the society shall have both a general strategy and instruments for handling adverse events, which may need timely action to prevent new cases and outbreaks. The strategy has an observational component and, in some aspects, a defensive or pro-active profile. However, in principle, this strategy may function and be efficient with or without the establishment of efficient control of the chemical ingredients of the inks.

ence any element of that action at any time when the process can be qualified, thus reducing the risk taken by the tattooed individual. This concept is inspired by seamless or integrated care, which has been successfully implemented in the health sector [23]. The concept of seamless prevention in tattooing is illustrated in figure 3 . The multi-target, preventive construction shall be active in every important cell, and there shall be no transitional friction or inconsistency and no defects in the range of executed preventive exercises as the customer moves from curiosity about tattooing, which is influenced by society, to interaction with the tattooist and finally to the longterm situation, which is when complications shall be limited and, if present, treated properly, diagnosed, registered and tracked backwards to learn and prevent similar occurrences from happening in the future.

As described previously in this chapter, the crucial points, or the 'engines', are the customer's decision to become tattooed and the act of tattoo- 
ing, i.e. the customer-tattooist interaction. Making this interaction as qualified and safety-oriented as possible is a core object of the seamless strategy.

The customer is influenced by the surrounding society and culture, with the present trendy promotion of tattooing and little public attention to safety issues. Public information and campaigns shall be instituted and make use of the most important media. The customer ultimately relies on close friends and relatives and his personal desires; however, both society and culture influence his decision.

The tattooist and customer interact at a level that may become intimate. If properly informed, in writing through an authorised brochure and verbally by the tattooist, about the limitations and risks of having a tattoo, the customer may decide not to be tattooed, which is the perfect form of prevention. However, the customer may of course decide to have the tattoo done after having the pros and cons being explained to him. Before giving his informed consent, there shall be an opportunity for a qualified ' $g o$ ' or 'no go'.

To do a good job, tattooists should practice ethical conduct, be qualified through formal education, and follow the laws and regulations. The tattooist and the tattoo parlour should be registered by a competent authority, which shall regularly inspect the facility to ensure that it is clean and suited for tattooing. The authority shall also ensure that the process of tattooing in the parlour, from $A-Z$, is up to the defined standards, including the proper use of the machines, needles and tattoo inks. The parlour shall have and follow an authorised hygiene standard that describes situations and sources that can expose the customer to risk of infection. The parlour shall also have a policy to prevent the tattooist himself from being infected by a customer through blood-borne viral agents. The parlour shall have a procedure for buying, maintaining and storing supplies and, if applicable, list the expiration date of the products. There shall be a defined procedure for pre-treat- ment and after-care that includes written information to the customer about the latter. The tattooist shall perform systematic filing of all relevant data, including precise data on the ink used, for the purpose of keeping a track record in the case of complications from tattooing for a particular customer. The tattooist shall on the day the tattoo is performed routinely supply the customer with a written record about the tattoo and the specific ink used, to be kept by the customer at home in his private files.

The suppliers of inks, tattoo needles and other utilities shall ensure that inks, needles and utilities are produced under good manufacturing standards and meet the highest achievable safety for a product for human use. This cannot be achieved unless there is a legal basis and standards. Needles and inks must be effectively sterile, with the method and date of sterilisation documented. Inks shall be delivered in unbroken and undamaged containers that are constructed to minimise contamination during use. The labelling of content, manufacturer, production date etc. shall follow requirements given by the relevant competent authority. The expiration date of inks is presently no more that a guess and some print on the product and is not documented in any way. In contrast, in the pharmaceutical industry, the expiration date and shelf life of a product is carefully measured and documented to become useful and reliable.

As mentioned above, competent authorities have a number of jobs related to the system and the daily business of tattoo parlours. Their role is to ensure that an appropriate system is established, implemented and followed.

Authorities have another important role, namely establishing a surveillance system for reporting clinical adverse events due to tattooing, especially medical events requiring treatment by medical health providers, clinics and hospitals. Such a system, to which customers, tattooists, clinics and hospitals may report, is needed to trace inks that pose a risk and other hazards, 
which need to be identified and handled without delay to minimise subsequent events and timely and efficiently eliminate the cause of problems. Such a system should be national and ideally involve European countries reporting on a European platform. A surveillance system is a major need since the chemical and particle safety of tattoo inks remain uncertain.

\section{Adverse Events from Tattooing and Their Medical Treatment and Service}

The tattoo boom is paralleled by adverse medical events that require medical diagnosis and treatment and are in line with any other significant disease that causes health problems. Sufferers are presently diffused among different specialties, such as general practice, dermatology, internal medicine, infectious medicine, general surgery, plastic surgery, laser surgery etc., and consequently may be seen as isolated cases. It is necessary to improve the diagnosis and treatment of complications from tattoos and register these complications case-by-case to generate experience, research and education that matches the present and future situation of the hundreds of millions of Europeans being tattooed and the hundred of thousands having complaints or complications specific for tattoos. Health care systems need to establish dedicated and qualified service to sufferers of tattoo complications, who are diseased and documented as burdened and disabled to the same degree as other patients who are offered public care [24]. Societies also need a knowledge reference in order to efficiently monitor the tattoo field.

\section{References}

1 Council on Health and Disease Prevention, Denmark. Tattooing, Health, Risks and Culture. Report under publication, 2015.

-2 De Cuyper C, Perez-Cotapos M-L: Dermatologic Complications with Body Art. Tattooos, Piercings and Permanent Make-Up. Berlin/Heidelberg, Springer Verlag, 2010.

-3 Kazandjieva J, Tsankov N: Tattoos: dermatological complications. Clin Dermatol 2007;25:375-382.

4 Kaatz M, Elsner P, Bauer A: Body-modifying concepts and dermatologic problems. Clin Dermatol 2008;26:35-44.

5 Kluger N: Cutaneous complications related to permanent decorative tattooing. Expert Rev Clin Immunonol 2010;6: 363-371.

6 Desai NA, Smith ML: Body art in adolescents: paint, piercings and perils. Adolesc Med 2011;22:97-118.

7 Wenzel SM, Rittman I, Landthaler M, Bäumler W: Adverse reactions after tattooing: review of the literature and comparison to results of a survey. Dermatology 2013;226:138-147.
8 Høgsberg T, Carlsen KH, Serup J: High prevalence of minor symptoms in tattooos among a young population tattooed with carbon black and organic pigments. J Eur Acad Dermatol Venereol 2013;27:846-852.

$\checkmark 9$ Hutton Carlsen K, Serup J: Photosensitivity and photodynamic events in balck, red and blue tattooos are common. A 'Beach Study'. J Eur Acad Dermatol Venereol 2013, DOI: 10.1111/jdv.12093.

10 Berchon E: Histoire Médicale du Tatouage. Paris, J-B Bailliè et Fils, 1869.

11 Klügl I, Hiller K-A, Landthaler M, Bäumler W: Incidence of health problems associated with tattooed skin: a nation-wide survey in German-speaking countries. Dermatology 2010;221:43-50.

$>12$ Kluger N, Koljonen V: Tattoos, inks and cancer. Lancet Oncol 2012;13:e161e168.

13 Pittelkow MR, Perry HO, Muller SA, Maughan WZ, O'Brian PC: Skin cancer in patients with psoriasis trteated with coal tar: a 25-year follow-up study. Arch Dermatol 1981;117:465-468.
14 Maughan WZ, Muller SA, Perry HO, Pittelkow MR, O'Brian PC: Incidence of skin cancers in patients with atopic dermatitis treated with coal tar: a 25-years follow-up study. J Am Acad Dermatol 1980;3:612-615.

15 The Danish Ministry of the Environment. Chemical substances in tattoo inks. Reports 215/216, 2012. ISBS 97887-92779-86-1. www.mst.dk.

16 Serup J, Hutton Carlsen K: Patch test study of 90 patients with tattoo reactions: negative outcome of allergy patch to baseline batteries and culprit inks suggests allergen(s) are generated in the skin through haptinization. Contact Dermatitis 2014;71:255-263.

17 Long GE, Rickman LS: Infectious complications of tattoos. Clin Infect Dis 1994;18:610-619.

18 Kluger N: Complications infectieuses cutanées assciées au tatouage permanent. Méd Mal Infect 2011;41:115-122.

19 Høgsberg T, Saunte DM, FrimodtMøller N, Serup J: Microbial status and product labelling of 58 original tattoo inks. J Eur Acad Dermatol Venereol 2013;27:73-80. 
20 Baumgartner A, Gautsch S: Hygienicmicrobiological quality of tattoo- and permanent make-up colours. J Verbrauch Lebensm 2011;6:319-325.

21 Laumann AE: History and epidemiology of tattoos and piercings, legislation in the United States; in: de Cuyoer C, Perez-Cotapos M-L (eds): Dermatologic Complications with Body Art. BerlinHeidelberg, Springer Verlag, 2010, pp $1-11$.
22 Council of Europe. Resolution ResAP(2008)1 on Requirements and Criteria for the Safety of Tattoos and Permanent Make-Up. Strasbourg, Council of Europe, 2008.
23 Thaldorf C, Liberman A: Integration of health care organizations: using the power strategies of horizontal and vertical integration in public and private health systems. Health Care Manag 2007;2:116-127.

24 Hutton Carlsen K, Serup J: Patients with tattoo reactions have reduced quality of life and suffer from itch: dermatology Life Quality Index and Itch Severity Score measurements. Skin Res Technol 2015, DOI: $10.1111 /$ srt 12164

Prof. Jørgen Serup, MD

Department of Dermatology D41, Bispebjerg University Hospital

Bispebjerg Bakke 23

DK-2400 Copenhagen NV (Denmark)

E-Mail Joergen.vedelskov.serup@regionh.dk 